\title{
Sequence Requirements for Transcriptional Arrest in Exon 1 of the Murine Adenosine Deaminase Gene
}

\author{
VIRARAGHAVAN RAMAMURTHY, ${ }^{1}$ MING-CHEI MAA, ${ }^{1}$ MICHAEL L. HARLESS, ${ }^{2}$ DAVID A. WRIGHT, ${ }^{2}$ \\ AND RODNEY E. KELLEMS ${ }^{1 *}$ \\ Verna and Marrs McLean Department of Biochemistry and Institute for Molecular Genetics, Baylor College of \\ Medicine, ${ }^{1}$ and Department of Molecular Genetics, M. D. Anderson Hospital Cancer Center,
} The University of Texas, ${ }^{2}$ Houston, Texas 77030

Received 18 October 1989/Accepted 3 January 1990

\begin{abstract}
We have previously shown that a transcription arrest site near the $5^{\prime}$ end of the murine adenosine deaminase (ADA) gene is significantly involved in the regulation of ADA gene expression. To facilitate the analysis of this transcription arrest site, we have analyzed the transcription products from cloned ADA gene fragments injected into Xenopus laevis oocytes. When genomic fragments spanning the $5^{\prime}$ end of the ADA gene were injected into oocytes, a 96-nucleotide (nt) ADA RNA was the major transcription product. The 5' end of this RNA mapped to the transcription initiation site for the ADA gene, and its $3^{\prime}$ terminus mapped 7 nt downstream of the translation initiation codon within exon 1. A 300-base-pair fragment of genomic DNA spanning the 5' end of the ADA gene was sufficient to generate the 96-nt transcript which accounted for approximately one-half of the transcription products from injected templates. Deletion of a segment of approximately 65 base pairs, located immediately downstream of the $3^{\prime}$ terminus of the 96-nt transcript, resulted in a substantial reduction in the synthesis of the 96-nt transcript and a corresponding increase in the production of larger transcripts. These studies show that the transcriptional apparatus of $X$. laevis oocytes responds to the transcription arrest site associated with exon 1 of the murine ADA gene and that oocyte injections provide a convenient functional assay for additional mechanistic studies.
\end{abstract}

The study of structural and functional controls exercised over transcription elongation and termination will help us to understand postinitiation processes in gene regulation. One of the obstacles in such an endeavor is the difficulty in discerning the steps involved in transcription per se and processing of the primary transcript. Transcription termination is still poorly defined in eucaryotic cells, mainly because the event is either preceded by, or is closely associated with, the processing of the $3^{\prime}$ end of the primary transcript (for a review, see references 6,23 , and 27). Intragenic transcription termination or a block to transcription elongation has been reported in a few cellular genes $(2,4,10,11)$ and viral genes $(14,18,22,28,31)$. This process may function to regulate tissue- or proliferation-specific expression of gene product(s), as in the case of c-myc $(4,26)$, c-myb (32), c-fos (11) and adenovirus late transcription (25), somewhat resembling attenuation in procaryotic transcription (for a review, see reference 35 ).

Adenosine deaminase (ADA) is an enzyme of purine metabolism that is present in all mammalian tissues. However, the levels of ADA protein and mRNA vary over a broad range, as much as 10,000 -fold in mice $(7,8)$. Recently, we and others have provided evidence for the existence of a transcription arrest site near the 5' end of the ADA gene and have shown that tissue-specific control of transcription elongation beyond the $5^{\prime}$ region plays a major role in regulating ADA gene expression $(8,21)$. Tissues with high levels of ADA protein and mRNA have an abundance of nascent ADA transcripts distributed throughout the 25-kilobase $(\mathrm{kb})$ ADA gene, whereas tissues with low levels of ADA protein and mRNA have abundant transcripts present only at the $5^{\prime}$ end of the gene (8). The use of a mouse fibroblast cell line with highly amplified copies of ADA genes enabled us to

* Corresponding author. localize the transcription block to within the first few hundred nucleotides of the ADA gene (M.-C. Maa et al., submitted for publication). However, the use of nuclear run-on experiments precludes the precise determination of the transcription block sites because of the limitations on the probe size that can be used for hybridizations. Further, as with c-myc, the presumed premature transcripts could not be detected in tissue culture cells. The Xenopus laevis oocyte transcription system, in contrast, has been successfully employed in the past to study nonpolyadenylated polymerase II transcripts (12). Bentley and Groudine (5) showed that the premature transcription termination of the c-myc gene could be characterized by using the Xenopus oocyte transcription system. In this regard, we have previously shown that injection of mouse ADA promoter-reporter gene fusion constructs into Xenopus oocytes results in faithful initiation of transcription from the ADA promoter (15). Hence, we have explored the utility of the Xenopus oocyte transcription system to analyze the premature transcription termination in ADA promoter-containing gene fragments. The major transcript from this region in injected oocytes was only 96 nucleotides (nt) long. In this manuscript, we have determined the template requirements for the efficient generation of this major transcript.

\section{MATERIALS AND METHODS}

Oocyte injection and RNA extraction. Stage VI oocytes were defolliculated, suspended in modified Barth's solution, and centrifuged to bring the nucleus to the surface; the nucleus was then injected with $4 \mathrm{ng}$ of supercoiled plasmid DNA in water. The injected oocytes were incubated for 22 to $26 \mathrm{~h}$ at $18^{\circ} \mathrm{C}$. RNA was extracted from five to seven pooled oocytes as described by Gurdon and Wickens (13). Total oocyte RNA was fractionated in $6 \%$ polyacrylamide- $8 \mathrm{M}$ urea, and gel fragments were cut out and extracted by 
suspension in $0.5 \mathrm{M}$ ammonium acetate-1\% sodium dodecyl sulfate-10 mM EDTA, followed by ethanol precipitation.

Northern (RNA) blot analysis. Two micrograms of total RNA from oocytes was fractionated by electrophoresis in $6 \%$ polyacrylamide gels with $8 \mathrm{M}$ urea, electroblotted onto a nylon membrane (Zetaprobe; Bio-Rad Laboratories), and UV cross-linked. The blots were prehybridized in $4 \times$ SSPE ( $1 \times \mathrm{SSPE}$ is $180 \mathrm{mM} \mathrm{NaCl}, 10 \mathrm{mM} \mathrm{NaH}{ }_{2} \mathrm{PO}_{4}$ [pH 7.4], and $1 \mathrm{mM}$ EDTA)-1\% sodium dodecyl sulfate- $0.5 \%$ nonfat milk and were hybridized in the same buffer containing a ${ }^{32} \mathrm{P}$ labeled oligonucleotide $(15)$ at $42^{\circ} \mathrm{C}$. The blots were washed in $0.5 \times \mathrm{SSC}(1 \times \mathrm{SSC}$ is $0.15 \mathrm{M} \mathrm{NaCl}$ plus $0.015 \mathrm{M}$ sodium citrate) $-0.5 \%$ sodium dodecyl sulfate at $65^{\circ} \mathrm{C}$ for $30 \mathrm{~min}$, dried, and exposed to X-ray films.

Nuclear run-on analysis. Germinal vesicles from oocytes injected with different plasmids were dissected with forceps in $25 \mathrm{mM}$ Tris ( $\mathrm{pH}$ 8)-10\% glycerol-5 $\mathrm{mM} \mathrm{MgCl}_{2}-2 \mathrm{mM}$ dithiothreitol. The isolated nuclei were washed once in nucleus storage buffer ( $50 \mathrm{mM}$ Tris [pH 8.0], $5 \mathrm{mM}$ magnesium acetate, $0.1 \mathrm{mM}$ EDTA, $10 \mathrm{mM}$ 2-mercaptoethanol, $15 \%$ glycerol). Nuclei from B-1/50 cells were isolated by suspending the cells in a solution containing $10 \mathrm{mM}$ Tris $(\mathrm{pH}$ 8.0), $10 \mathrm{mM} \mathrm{NaCl}, 3 \mathrm{mM} \mathrm{MgCl}{ }_{2}$, and $0.5 \%$ Nonidet $\mathrm{P}-40$ and were collected by centrifugation at $500 \times g$ at $4^{\circ} \mathrm{C}$. Nuclear run-on transcription with $\left[{ }^{32} \mathrm{P}\right] \mathrm{GTP}$ is described by Chinsky et al. (8). The labeled RNA was extracted as described by Chomczynski and Sacchi (9), hybridized to restriction fragments of appropriate plasmids electrophoresed in $0.8 \%$ agarose gels, and transferred to a nylon membrane (Zetaprobe; Bio-Rad). Hybridization was carried out for $36 \mathrm{~h}$ at $65^{\circ} \mathrm{C}$. The hybridized membranes were washed and prepared for autoradiography as described for Northern blot analysis.

Primer extension analysis. A synthetic oligonucleotide primer complementary to the mouse ADA transcript from the nontranslated leader region -53 to $-77(15)$ was labeled with $\left[\gamma^{32} \mathrm{P}\right] \mathrm{ATP}$ and hybridized to RNA at $72^{\circ} \mathrm{C}$ for $1 \mathrm{~h}$ in 10 $\mu l$ of hybridization solution $(0.3 \mathrm{M} \mathrm{NaCl}, 10 \mathrm{mM}$ Tris $[\mathrm{pH}$ 7.5], 1 mM EDTA). After hybridization, Tris, dithiothreitol, $\mathrm{MgCl}_{2}, 2 \mu \mathrm{l}$ of each of the four deoxynucleoside triphosphates $(10 \mathrm{mM}), 2 \mu \mathrm{l}$ of actinomycin $\mathrm{D}(400 \mu \mathrm{g} / \mathrm{ml}), 10 \mathrm{U}$ of reverse transcriptase, and water to $100 \mu \mathrm{l}$ were added and incubated at $37^{\circ} \mathrm{C}$ for $30 \mathrm{~min}$. The extended products were ethanol precipitated and fractionated on $8 \%$ polyacrylamide$8 \mathrm{M}$ urea gels.

Nuclease protection analysis. Probes for analyses were prepared by kinase reactions ( $5^{\prime}$ ends) or Klenow fill-in reactions $\left(3^{\prime}\right.$ ends) of isolated DNA fragments or in vitro transcription reactions with T7 RNA polymerase (uniformly labeled; 24). Total RNA from oocytes $(1 \mu \mathrm{g})$ or from tissue culture cells $(10 \mu \mathrm{g})$ was mixed, coprecipitated with 20,000 cpm of end-labeled DNA probes or $600,000 \mathrm{cpm}$ of uniformly labeled RNA probes, dissolved in $13 \mu$ l of $80 \%$ formamide$0.2 \mathrm{M} \mathrm{NaCl}-40 \mathrm{mM}$ piperazine- $N, N^{\prime}$-bis(2-ethanesulfonic acid (PIPES) (pH 6.8)-1 mM EDTA, denatured at $90^{\circ} \mathrm{C}$ for 5 min, and hybridized at $52^{\circ} \mathrm{C}$ for $3 \mathrm{~h}$. With DNA probes, the samples were digested in $0.3 \mathrm{ml}$ of $\mathrm{S} 1$ digestion buffer $(0.28$ $\mathrm{M} \mathrm{NaCl}, 30 \mathrm{mM}$ sodium acetate, $4.5 \mathrm{mM}$ zinc acetate, 1,000 $\mathrm{U}$ of $\mathrm{S} 1$ nuclease per $\mathrm{ml}$ [Pharmacia]) at $37^{\circ} \mathrm{C}$ for $45 \mathrm{~min}$. With the RNA probe, the hybridization was carried out at $44^{\circ} \mathrm{C}$ and the samples were digested in $0.3 \mathrm{ml}$ of RNase buffer $(0.3 \mathrm{M} \mathrm{NaCl}, 10 \mathrm{mM}$ Tris [pH 8.0], $5 \mathrm{mM}$ EDTA, $80 \mu \mathrm{g}$ of RNase A per ml, $40 \mathrm{U}$ of RNase T1 per ml) at $24^{\circ} \mathrm{C}$ for 60 min. The nuclease-resistant fragments were separated by electrophoresis in $6 \%$ polyacrylamide gels with $8 \mathrm{M}$ urea.

Plasmid constructions. Plasmids E1.4 and X6.4 were subcloned from genomic cosmids obtained from B-1/50 cells
(15). Deletion mutants $1.2,0.7$, and 0.4 were obtained by ExoIII digestion, mung bean nuclease cleavage, and blunt ends recircularization. Deletion mutants $\Delta 1$ (BsmI-SmaI), $\Delta 2$ (BsmI-NarI), $\Delta 3$ (BsmI-BalI), $\Delta 4$ (BsmI-SmaI of polylinker), and 0.3 (SmaI-SmaI of polylinker) were obtained by restriction with respective enzymes, $\mathrm{S} 1$ digestion, and recircularization. All constructs were sequenced to ascertain the extent of the deletions. The minigenes were constructed by inserting a 2.8-kb HindIII fragment at the HindIII site of the ADA cDNA and inserting a 5.4- or a $2.7-\mathrm{kb} 5^{\prime}$ flanking fragment containing the ADA promoter at the NcoI site (D. E. Ingolia, Ph.D. dissertation, Baylor College of Medicine, Houston, Tex., 1987). The nco mutant was constructed by restricting E1.4 with NcoI, filling sticky ends with Klenow, and religating the ends. The $1+9$ fusion was constructed by inserting a $0.65-\mathrm{kb} \mathrm{Bam} \mathrm{HI}$ fragment containing part of exon 9 and exons 10 and 11 at the $N c o$ I filled-in end of E1.4.

\section{RESULTS}

Detection of short ADA transcripts from injected $X$. laevis oocytes. Transcriptional analysis of the mouse ADA locus indicated that the transcription complexes along the gene were not randomly distributed and arrested predominantly within the first $200 \mathrm{nt}$ of the transcription unit (8; Maa et al., submitted). These aborted transcripts are presumably nonpolyadenylated and are, hence, unstable in mammalian cells. Since nonpolyadenylated transcripts appear to be more stable in Xenopus oocytes $(5,12)$, we wanted to determine whether the transcription of ADA gene fragments could result in the generation of short transcripts less than $200 \mathrm{nt}$ long. Figure 1 depicts the exon-intron organization of the ADA gene and the location of two genomic subclones from the ADA locus used in this study. These clones, X6.4 and E1.4 (a subclone of X6.4), were injected into oocytes, and RNA isolated $24 \mathrm{~h}$ later was analyzed by Northern blot hybridization. The blots were probed with a radiolabeled oligonucleotide complementary to the $5^{\prime}$ nontranslated region of mouse ADA transcripts ( -53 to $-77 \mathrm{nt}$, with respect to the translation start site; 14). The probe did not hybridize to the RNA from uninjected oocytes (Fig. 1, lane 1) but hybridized to RNA from oocytes injected with mouse ADA gene subclones (Fig. 1, lanes 2 and 3), indicating that the probe was specific for mouse ADA transcripts. The size distribution of mouse ADA-specific RNA from oocytes injected with the two different genomic subclones was identical. A major transcript of $96 \mathrm{nt}$ long was detected. Lower levels of a few other short transcripts (a doublet around 105 and $196 \mathrm{nt}$ ) were also consistently detected. These results indicated that short ADA-specific transcripts accumulated in oocytes and that the additional sequences contained in X6.4 (i.e., the $0.65-\mathrm{kb}$ sequence in the $5^{\prime}$ flanking side and the 4.4-kb sequence of the first intron) did not alter the pattern of stable ADA-specific transcripts. Thus, the DNA sequence contained in the smaller plasmid, E1.4, was sufficient to generate short 96-nt mouse ADA-specific transcripts, when injected into Xenopus oocytes.

Characterization of ADA transcripts from injected oocytes. To determine if the transcripts observed in Fig. 1 from injected oocytes were initiating at appropriate sites, S1 nuclease protection and primer extension analysis were performed (Fig. 2). S1 nuclease protection analysis indicated that the 5' ends of RNA from oocytes injected with X6.4 or E1.4 were identical, 89 and 90 nt upstream of the translation start site (Fig. 2A, lanes 2 and 4). These specific fragments 


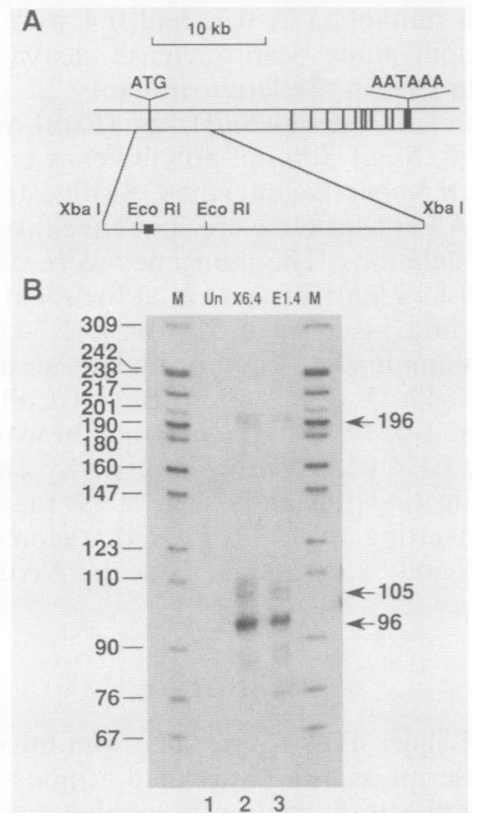

FIG. 1. Northern blot analysis of transcription products from injected $X$. laevis oocytes. (A) Exon-intron organization of the murine ADA gene and the region from where the two subgenic clones X6.4 and E1.4 were isolated. The solid boxes indicate exons. (B) Northern blot analysis of stable transcripts from oocytes. RNA $(2 \mu \mathrm{g})$ from uninjected oocytes (Un) or oocytes injected with X6.4 or E1.4 plasmids was fractionated in a $6 \%$ polyacrylamide $-8 \mathrm{M}$ urea gel and electroblotted onto a nylon membrane. The blot was probed with a 25-nt murine ADA-specific probe. The size markers are pBR322 digested with $M s p I(M)$. The sizes of the transcripts are indicated.

were not protected in RNA from oocytes coinjected with $\alpha$-amanitin, but a small amount of $\alpha$-amanitin-insensitive transcription could be seen (Fig. 2A, lanes 1 and 3). These results suggest that the transcripts with $5^{\prime}$ termini 89 to $90 \mathrm{nt}$ upstream of the translation start site are polymerase II products. To compare ADA promoter-derived transcription in injected oocytes with transcription in mouse cells, primer extension analysis of RNA from various sources was performed. As described previously (15), ADA-specific RNA from B-1/50 cells initiated at multiple sites (Fig. 2B, lane 4). This was also true for RNA from B-1/150 cells (Fig. 2B, lane 5 ) in which the ADA genes are amplified to a higher level (16). In oocytes injected with E1.4, the preferred initiation sites were at -89 and $-90 \mathrm{nt}$. This was not an anomaly, since in mouse tissues with high ADA mRNA levels, such as the tongue, the initiation sites mapped from -89 to $-90 \mathrm{nt}$ (Fig. 2B, lane 6; 8). In this respect, transcription of the ADA sequence in oocytes resembled transcription of the gene in mouse tissues. These data showed that the $5^{\prime}$ ends of the ADA-specific transcripts in the injected oocytes correspond to the ADA transcription initiation site at -89 to $-90 \mathrm{nt}$ and suggest that the ADA promoter element is properly recognized by the oocyte transcription system.

S1 nuclease analysis was performed to determine the $3^{\prime}$ terminus of the major transcript (96 nt) and the extent of transcription readthrough past this site in injected oocytes (Fig. 3A). A 96-nt transcript whose 5' end maps from -89 to $-90 \mathrm{nt}$ from the translation start site should have its $3^{\prime}$ end at $+7 \mathrm{nt}$, provided there was no splicing involved in the generation of the transcript. Thus, a probe from the DdeI

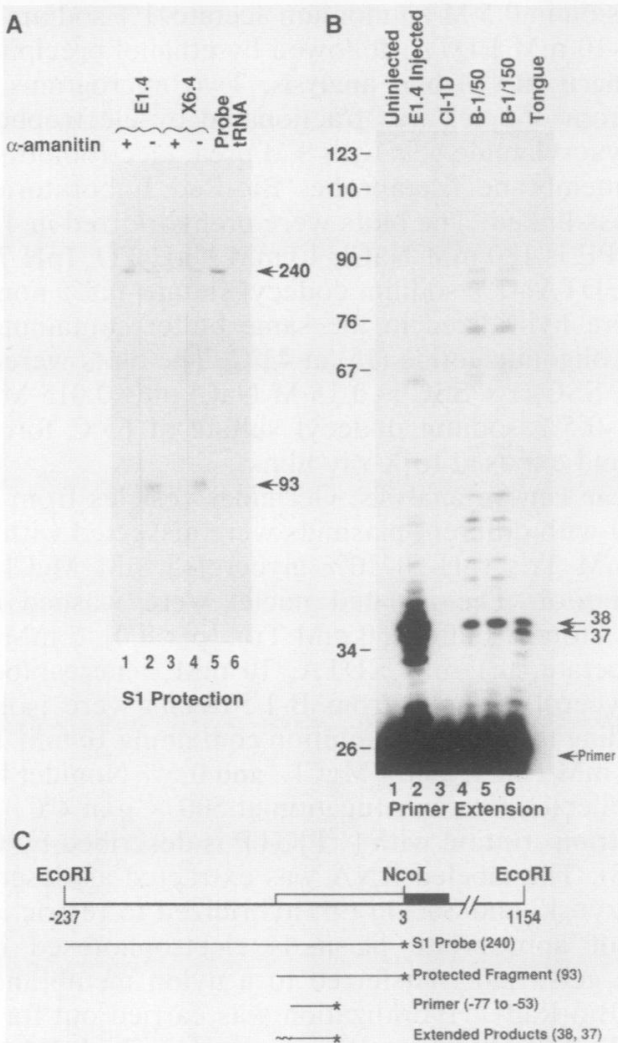

FIG. 2. Determination of the initiation sites of ADA transcripts (A) S1 nuclease protection analysis. The 240-nt EcoRI-NcoI fragment from E1.4 was 5' end labeled at the Ncol site and hybridized to total RNA from oocytes injected with E1.4 or X6.4 in the presence $(+)$ or absence $(-)$ of $\alpha$-amanitin $(2 \mu \mathrm{g} / \mathrm{ml})$ or to yeast tRNA. The mixture was digested with $\mathrm{S} 1$ nuclease, and the protected fragments were separated in a $6 \%$ polyacrylamide $-8 \mathrm{M}$ urea gel. The sizes of the protected fragment and the undigested probe are indicated. (B) Primer extension analysis. A 5'-end-labeled oligonucleotide corresponding to the complement of the ADA transcript $(-53$ to -77$)$ was hybridized to total RNA from uninjected oocytes, oocytes injected with plasmid E1.4, mouse fibroblast cells $\mathrm{C} 1.1 \mathrm{D}, \mathrm{B}-1 / 50$, or $\mathrm{B}-1 / 150$, or mouse tongue. The primer was extended with reverse transcriptase, and the products were separated in a $8 \%$ polyacrylamide- $8 \mathrm{M}$ urea gel. The sizes of the extended products are marked with arrows, and the locations of size markers are indicated. (C) Map of the plasmid E1.4 with the locations of the S1 probe, the fragment protected from the probe, the primer, and its extension products (wavy line) are depicted. The open and the closed boxes indicate the nontranslated and the translated part of exon 1

end (-86 nt) should protect a 93-nt fragment in a nuclease protection assay. A 93-nt fragment was indeed protected, indicating that no splicing was involved in the generation of the major transcript. The minor transcript of $105 \mathrm{nt}$ (Fig. 1) protected a fragment of $102 \mathrm{nt}$. All transcripts past the SmaI site protected a fragment of $165 \mathrm{nt}$. The intensity of this fragment, which represents readthrough past the SmaI site, was about the same as that of the 93-nt protected fragment, suggesting that about one-half of the transcripts end at +7 to generate the 96-nt RNA.

To ascertain that the major transcripts seen on Northern blots (Fig. 1) and by end mapping (Fig. 3A) were identical, total oocyte RNA was fractionated in a denaturing acrylamide gel and two fractions (fraction 1, 125 to $175 \mathrm{nt}$; 


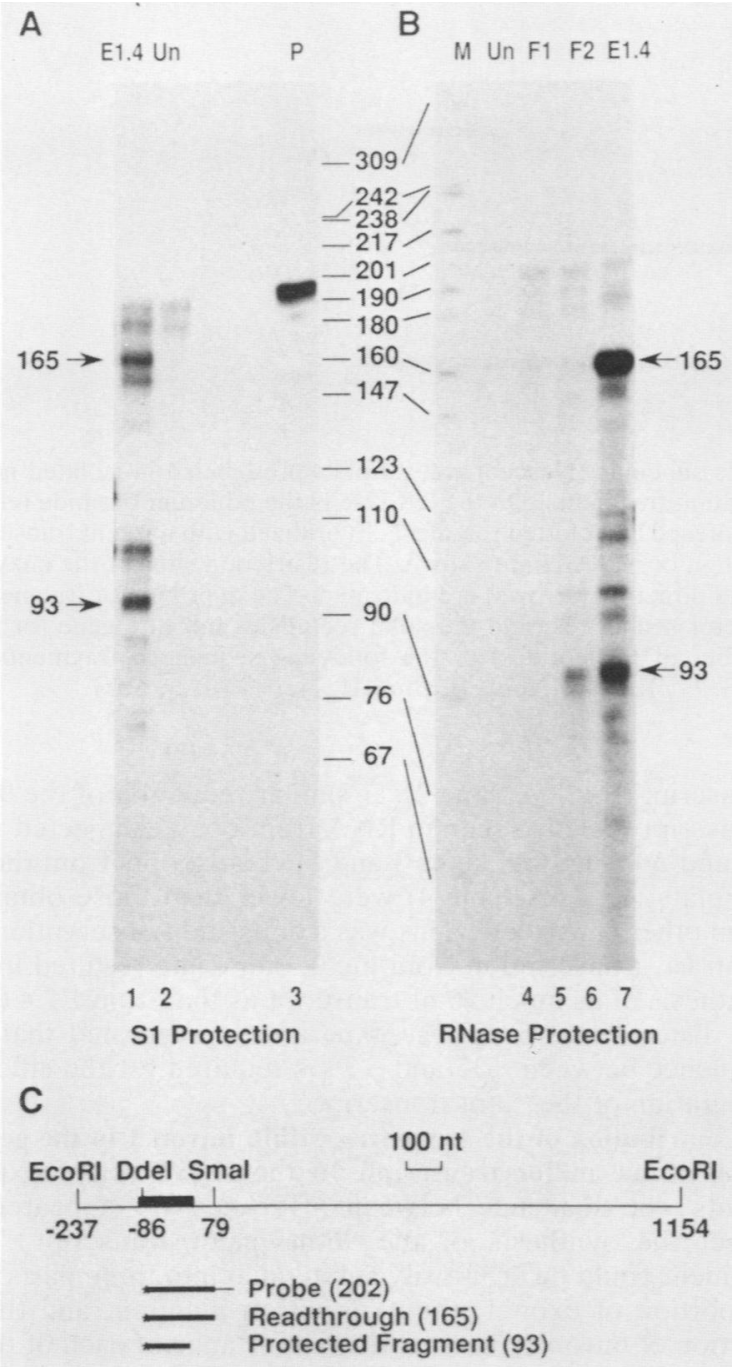

FIG. 3. Determination of the 3 ' ends of transcripts from oocytes. (A) S1 protection analysis. The 202-nt fragment containing the DdeI-SmaI region was $3^{\prime}$ end labeled at the DdeI site (P) and hybridized to total RNA from uninjected (Un) or E1.4-injected oocytes (E1.4). The mixtures were digested with S1 nuclease, and the protected fragments were separated in a $6 \%$ polyacrylamide $-8 \mathrm{M}$ urea gel. Locations of the size markers are shown. (B) RNase protection analysis. Gel-purified RNA from E1.4-injected oocytes was eluted from $6 \%$ polyacrylamide $-8 \mathrm{M}$ urea gel as fraction 1 (125 to $175 \mathrm{nt}$ ) and fraction 2 (75 to $125 \mathrm{nt})$. An RNA molecule complementary to the sequence between DdeI and SmaI was generated with T7 RNA polymerase and was hybridized to RNA from uninjected oocytes (Un), fraction 1 (F1), fraction 2 (F2), or total RNA from injected oocytes (E1.4). The mixtures were digested with RNase $A$ and $R$ Nase $T_{1}$, and the protected fragments were separated in a $6 \%$ polyacrylamide $-8 \mathrm{M}$ urea gel. Locations of the size markers are shown. The size markers (M) are MspI-cut pBR322. (C) Map of the plasmid E1.4 with the locations of relevant restriction sites. The solid box indicates exon 1 . The thick line represents the sequence from the ADA gene, the thin line represents the plasmid vector sequence, the asterisk indicates the labeling site. and the numbers indicate the length (in nucleotides).

fraction 2, 75 to $125 \mathrm{nt}$ ) were extracted and subjected to RNase protection analysis (Fig. 3B). A 93-nt RNase-resistant fragment was protected from the total oocyte RNA (Fig. 3B, lane 7) and from fraction 2 (Fig. 3B, lane 6) only. This confirmed that the major transcript observed in blots has its $3^{\prime}$ terminus at +7 . Also, these experiments confirmed that there was a minor ADA transcript with its $3^{\prime}$ terminus mapping to +16 .

Transcription of the ADA gene in murine fibroblasts and $X$. laevis oocytes is similar. The above-mentioned experiments showed that the majority of the ADA transcripts synthesized in injected oocytes are very short and, hence, predict that transcription at the ADA locus must terminate very near the first exon. To test this prediction, we compared the transcription of an injected ADA gene fragment (E1.4) with that of the complete ADA gene in a mouse fibroblast cell line with amplified copies of the gene $(B-1 / 50 ; 16)$. Labeled nascent transcripts from nuclear run-on reactions were hybridized to fragments of E1.4 generated by EcoRI and NdeI digestions, which allows for the comparison of the transcriptional activity within the promoter-proximal and the promoter-distal segments of E1.4 (Fig. 4). Transcription of the ADA locus in B-1/50 nuclei was greater in the promoter-proximal half (fragment II) than in the promoter-distal half (fragment III). These results were consistent with the data obtained with single-strand DNA probes (Maa et al., submitted). In oocytes injected with E1.4, a strong transcriptional signal was observed in the promoter-proximal sequence (fragment II), but only a very weak signal was detected from the promoter-distal sequence (fragment III). Hybridization to the plasmid vector sequence (fragment I) suggested that the opposite strand might be transcribed from the ADA promoter, consistent with the observations of Maa et al. (submitted). Alternatively, transcription could initiate within the plasmid vector sequence and represent a nonspecific background of transcription initiation in this system. Transcription from the plasmid vector sequence has been observed in $\alpha$-amanitin-pretreated oocytes as well (Z. Chen and R. E. Kellems, unpublished observations), suggesting that other RNA polymerases contribute to the transcription signal. Indeed, transcripts from plasmid vector sequences in injected oocytes have been observed by other investigators (5). Notwithstanding transcription within the plasmid vector sequence in injected oocytes, these results suggest that the transcription patterns of the ADA gene fragment E1.4 in the oocytes and the 25-kb ADA gene in the B-1/50 nuclei are similar.

To ensure that the asymmetric distribution of RNA polymerase complexes along E1.4 was unique to the ADA gene fragment tested and was not due to some insufficiency of the Xenopus oocyte transcription system, we examined the transcription of two other genes injected into oocytes as plasmids. In separate experiments, oocytes were injected with pTKGH, which contains the human growth hormone gene driven by the herpes simplex virus-thymidine kinase promoter, or the 5.4 minigene, in which the ADA cDNA is driven by the ADA promoter. Labeled nascent transcripts from these oocytes were hybridized with gene fragments representing promoter-proximal and promoter-distal segments (Fig. 4). The promoter-proximal and promoter-distal segments of both genes showed equivalent hybridization signals, indicating a uniform distribution of RNA polymerase complexes. These results suggest that only with E1.4 was the progression of RNA polymerase complexes along the template impeded when injected into oocytes.

Transcripts from deletion mutants. To determine the sequence requirements contributing to the generation of the major 96-nt transcript in oocytes, two series of deletion mutants were constructed. In the first series, sequences from the $3^{\prime}$ end of plasmid E1.4 were deleted up to $+79 \mathrm{nt}$ from 

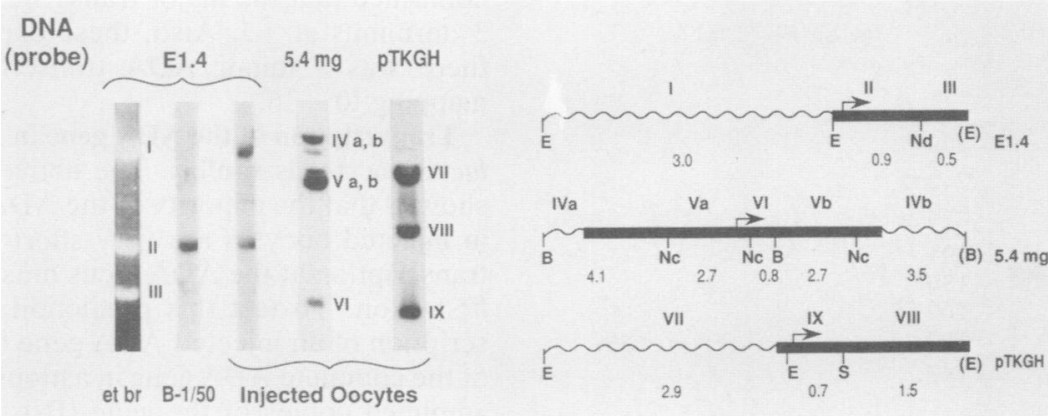

FIG. 4. Nuclear run-on analysis of transcription of the ADA gene and its subclone. Nascent transcripts were labeled in isolated nuclei (B-1/50 cells) or in dissected germinal vesicles and were hybridized to restriction fragments. On the left side is the ethidium bromide (et br)stained gel of restriction fragments for E1.4. Autoradiograms of the electrophoresed and blotted fragments hybridized with nascent transcripts from B-1/50 nuclei, germinal vesicles injected with E1.4, 5.4 minigene $(5.4 \mathrm{mg})$, or pTKGH are shown. The restriction sites of the enzymes used, the sizes of the fragments (in kilobases), and the transcription start and direction (arrows) are indicated. The migration of fragments 1 through IX is indicated by the bands. The wavy lines indicate plasmid vector sequences, and the solid rectangles indicate gene (or gene fragment) sequences. The number of guanine nucleotides (and total number of nucleotides) of the following sequenced fragments are provided: II, 316 (875); III, 152 (520); VIII, 416 (1584); and IX, 228 (774). E, EcoRI; Nd, NdeI; B, BamHI; Nc, NcoI; S, SacI.

the translation start codon. This series of deletions did not extend into the part of E1.4 coding for the 96-nt transcript. The various deletion clones were injected into oocytes, and stable transcripts were analyzed by Northern blotting and hybridization with a ${ }^{32}$ P-labeled oligonucleotide probe (Fig. 5). The generation of the 96-nt transcript was not significantly affected in any of the clones, indicating that the sequences deleted in this series (from +79 to +1154 ) were not required and that $0.3 \mathrm{~kb}$ of the $\mathrm{ADA}$ sequence was sufficient for its generation.

In the second series, sequences were deleted from the BsmI site in exon 1 (Fig. 6B). All of these constructs, however, retained the ADA promoter and about $110 \mathrm{nt}$ of exon 1 , including the coding sequence for the 96-nt transcript. The various constructs were injected into oocytes, and RNA recovered $24 \mathrm{~h}$ postinjection was analyzed (Fig. 6A). Deletion of the sequence between +14 and +80 in $\Delta 1$ resulted in a significant (10- to 12 -fold) reduction of the $96-\mathrm{nt}$ transcript (Fig. 6A, lane 2). A similar reduction of the 96-nt transcript was also seen in RNA from oocytes injected with $\Delta 2$ and $\Delta 4$ (Fig. 6A, lanes 3 and 5). Transcripts from the $\Delta 3$ template (Fig. 6A, lane 4) were fewer than those obtained from other constructs. This was a consistent observation. In contrast, transcription from the 0.3 template resulted in the synthesis of as much 96-nt transcript as that from E1.4 (Fig. 6A, lanes 1 and 6). These experiments suggested that the sequence between +15 and +79 is required for the efficient generation of the 96-nt transcript.

Contribution of the sequences within intron 1 to the generation of the major transcript. In the above set of experiments, the sequence between +15 and +79 appeared to affect the synthesis of the 96-nt major transcript. This segment could be arbitrarily subdivided into three parts: the $3^{\prime}$ portion of exon 1 , the exon-intron junction, and the $5^{\prime}$ portion of intron 1 . The relative importance of each of these sequences to the generation of the 96-nt transcript was
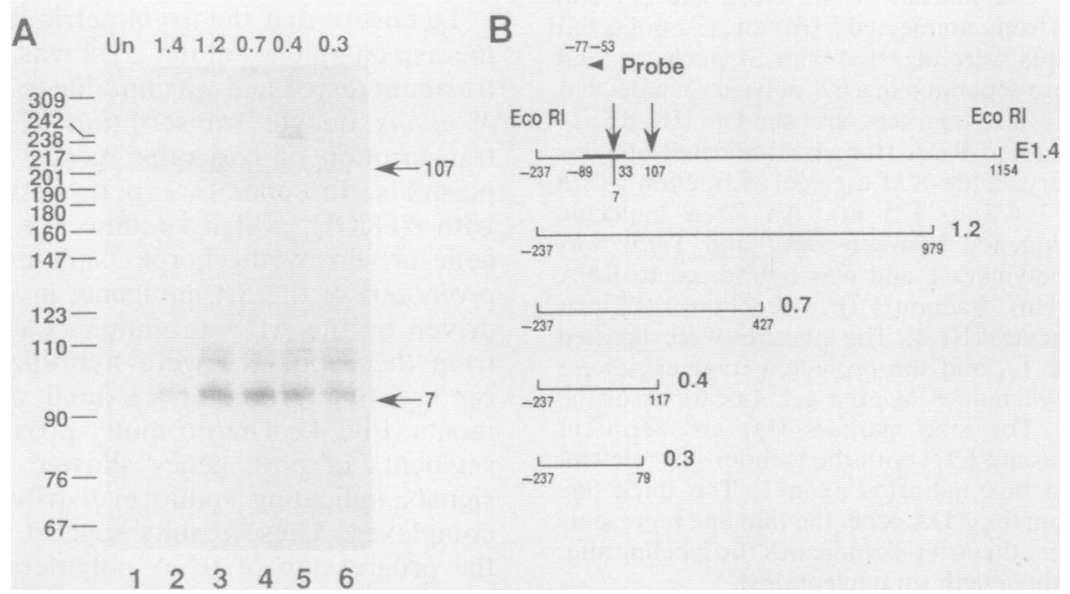

FIG. 5. Northern analysis of transcription products from the $3^{\prime}$ deletion mutants. (A) Equal amounts ( $\left.2 \mu \mathrm{g}\right)$ of RNA were used in each lane. The analysis was performed as described in the legend to Fig. 2. The template injected to generate the RNA in each lane is indicated on top; RNA from uninjected oocytes (Un). The locations of the DNA size markers are shown. The major transcripts from oocytes are identified by the location of their 3' termini. (B) Map of E1.4 with reference to the translation start site. Exon 1 is depicted as a thick line. The arrows point to the $3^{\prime}$ ends of the major transcripts in the injected oocytes. The location of the oligonucleotide probe and the sequence retained in the deletion mutants and their nucleotide endpoints are also indicated. 
A

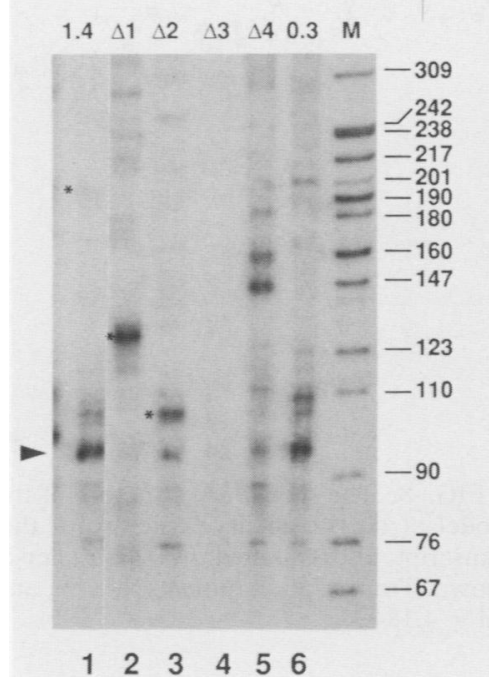

123456
B

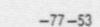

$-77-53$
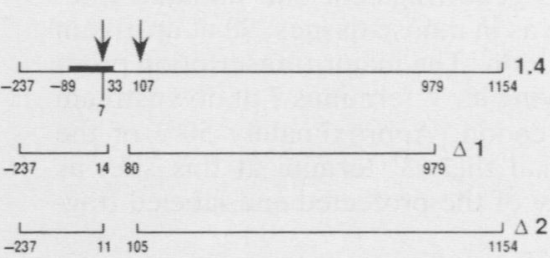

$-237$
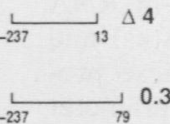

FIG. 6. Northern analysis of transcription products from the internal deletion mutants. (A) Equal amounts ( $2 \mu g$ ) of RNA were used in each lane. The analysis was performed as described in the legend to Fig. 2 . The template injected to generate the RNA in each lane is indicated on top. The location of the size markers and the major transcription product from oocytes ( $96 \mathrm{nt}$ long, terminating at +7$)$ is indicated by an arrowhead. The asterisks indicate the location of the 196-nt transcript (terminating at +107 ) migrating as smaller molecules in $\Delta 1$ and $\Delta 2$ due to deletion of internal sequences. (B) Map of E1.4 with reference to the translation start site. Exon 1 is depicted as a thick line. The arrows point to the $3^{\prime}$ ends of the major transcripts in the injected oocytes. The location of the oligonucleotide probe and the sequence retained in the deletion mutants and their nucleotide endpoints are also indicated.

examined. By using an ADA minigene, which lacks intron 1, we could address whether the sequence contained in exon 1 (and not intron 1) was sufficient for the generation of the 96-nt transcript. The 96-nt transcript was generated from the ADA minigene injection into oocytes; however, like $\Delta 1$, only low levels of the 96-nt transcript were observed (Fig. 7, lanes 2 and 3 ). Thus, even though the sequence encompassing the

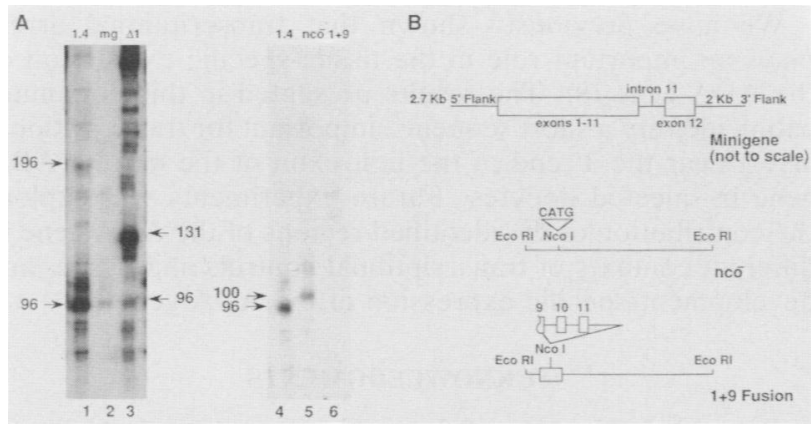

FIG. 7. Contribution of intron 1 sequences for the generation of the major ADA transcript in oocytes. (A) Northern analysis was performed as described in the legend to Fig. 2. The sizes of the various transcripts are indicated. Deletion of $65 \mathrm{bp}$ in $\Delta 1$ reduces the 196-nt transcript of E1.4 to $131 \mathrm{nt}$ in $\Delta 1$. Insertion of $4 \mathrm{bp}$ in the nco mutant increases the 96-nt E1.4 to $100 \mathrm{nt}$ in the nco mutant. The various recombinant constructs used in this experiment are indicated in panel $\mathrm{B}$. The minigene has $2.7 \mathrm{~kb}$ from the $5^{\prime}$ flanking sequence, exons 1 through 11 from the cDNA, intron 11 , exon 12 , and $2 \mathrm{~kb}$ of the $3^{\prime}$ flanking sequence. The nco mutant plasmid was constructed by inserting $4 \mathrm{nt}$ at the NcoI site. The $1+9$ fusion plasmid was constructed by inserting a 0.65 -kb genomic fragment containing the $3^{\prime}$ portion of exon 9 , exon 10 , and exon 11 at the $N c o$ I site. promoter and first exon was sufficient for generation of the 96-nt transcript, the sequence in the 5 ' region of intron 1 from +34 to +79 was necessary for optimal generation of this transcript.

To determine whether the exon-intron junctional motif alone was sufficient for the generation of the short transcript, we replaced the exon-intron junction of exon 1 with that of exon 9 . The $0.65-\mathrm{kb}$ genomic fragment from the ADA locus containing the $3^{\prime}$ parts of exon 9 , exon 10 , and exon 11 was inserted at the NcoI site $(+4)$ of E1.4. The size of the 3' portion of exon 9 ( 29 base pairs [bp]) was similar to the size of exon 1 (30 bp), which was replaced. No 96-nt-long transcript was detected by the transcription of this construct in oocytes (Fig. 7, lane 6). This suggested that an exon-intron junction is not solely responsible for, or alternatively that the inserted sequence prevents the generation of, the 96-nt transcript. The 4-bp insertion mutant (nco mutant) generated by $N$ coI cleavage, Klenow fill-in, and ligation served as a preinsertion control for the exon fusion construct. Transcription of the nco mutant generated a major product of 100 nt (Fig. 7, lane 5). Thus, insertion of $4 \mathrm{nt}$ within the coding template at the $N$ col site did not affect the generation of the major transcript.

\section{DISCUSSION}

The experiments reported here showed that the transcription system of $X$. laevis oocytes is able to recognize a transcription arrest site near the $3^{\prime}$ end of the first exon of the murine ADA gene. Transcription of the gene fragment E1.4 in Xenopus oocytes was similar to transcription of the corresponding region in the amplified ADA genes of the mouse fibroblast cells B-1/50, i.e., the promoter-proximal segment showed strong transcriptional activity while the promoter-distal segment showed weak transcriptional activity. However, unlike the case in mammalian cells, the short 
transcripts accumulate in the oocytes, and this has allowed us to characterize their sizes and locations. The Xenopus oocyte transcription system recognized the promoter element within the injected gene fragment and initiated transcription at the same site as in mouse tissues, $90 \mathrm{nt}$ upstream of the translation start codon. The major transcription product was a 96-nt RNA having its $3^{\prime}$ terminus 7 nt downstream of the translation start codon. Approximately $50 \%$ of the transcription products had their $3^{\prime}$ termini at this site, as indicated by the intensity of the protected end-labeled fragments.

The ease of following the transcription products from injected oocytes has facilitated the study of the sequence requirements for the generation of the 96-nt RNA. We assume that the variations in the level of the 96-nt RNA resulted from the transcription-associated process(es) and not from stability differences, and we refer to these processes as transcription termination. Another parameter which could affect generation of the short transcripts in an unknown way is transcription from the plasmid vector sequences. We consider the contribution from transcription of the vector sequences to the generation of the 96-nt transcript to be minimal, since the efficiency of generating this short transcript was not significantly affected from different plasmids containing vector sequence from 32 (in X6.4) to $91 \%$ (in 0.3 ) of the plasmid sequences. Just $0.3 \mathrm{~kb}$ of the $5^{\prime}$ region of the ADA gene, including the promoter element, was sufficient for the efficient generation of this transcript. Analysis of internal deletion mutants revealed that the sequence from +15 to +79 is necessary for the efficient generation of the 96-nt transcript, since removal of this sequence resulted in a poor utilization of the termination site. The poor utilization of this transcription termination site following mutational alterations was accompanied by utilization of a succeeding termination site, normally at +196 , but which had been moved closer to the transcription initiation site in $\Delta 1$ and $\Delta 2$ mutants because of deletions of 65 and $93 \mathrm{nt}$, respectively. Thus, poor utilization of the first termination site resulted in increased transcription readthrough and a concomitant utilization of succeeding termination sites. This suggests that the ADA transcription complex may be inherently unstable and may pause or terminate transcription when the complex encounters any of the redundant termination signals, or perhaps even cryptic termination signals.

In other genes in which structural requirements for premature transcription termination have been studied, the necessary sequences were completely within the template sequence of the premature transcript $(17,19,29,30,33)$. In this respect, mechanistically, the transcriptional arrest in the ADA gene may be somewhat different from these examples. However, there is some precedence for signals $3^{\prime}$ to the ends of transcripts which affect cotranscriptional events. In the case of $5 \mathrm{~S}$ and ribosomal RNA gene transcription, the steady-state RNA is generated by terminating transcription downstream of the actual 3' terminus, followed by nucleolytic cleavage $(20,34)$. By analogy, the generation of the 96-nt transcript may be related to a cotranscriptional or posttranscriptional processing. We are currently investigating these possibilities.

When analyzed by in vitro transcription reactions, the sequence determining efficient termination depended on the ability of the RNA to form stable stem-loop structures or on the presence of consecutive runs of four or more $T$ residues in the DNA or both (3). An inspection of the sequence around the premature transcription termination site in the ADA gene shows that there are only very short runs of

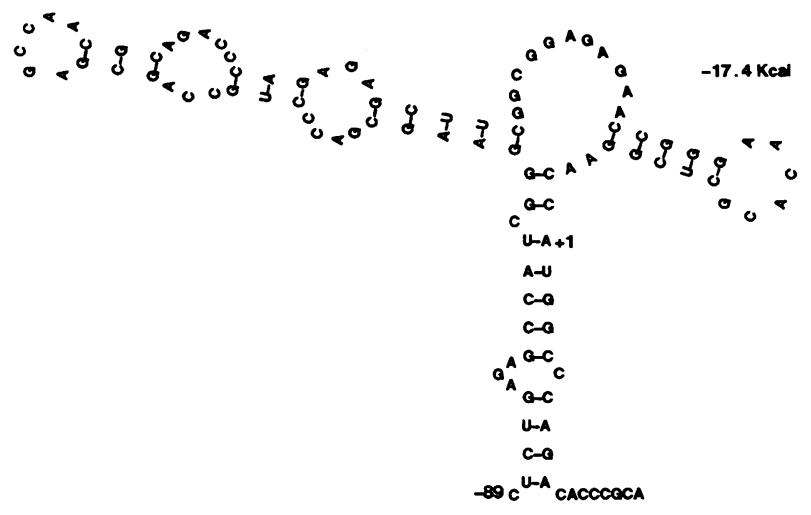

FIG. 8. The secondary structure of the ADA short transcript. A model of the secondary structure of the first $107 \mathrm{nt}$ of the ADA transcript, as predicted by the Zuker-Stiegler algorithm (36), is shown. Transcription initiates at -89 , and translation is from +1.1 $\mathrm{cal}=4.184 \mathrm{~J}$

homopolymers (three or four) and no more than three consecutive $T$ residues (Maa et al., submitted). In the case of $c-m y c$, although there were runs of $\mathrm{T}$ in both the human and the mouse gene, deletion of these sequences did not affect the use of the transcription termination sites (5). Examination of the first $107 \mathrm{nt}$ of the ADA transcript suggests that it has a capacity to attain a stable secondary structure. A model of the secondary structure as predicted by the ZukerStiegler algorithm (36) is depicted in Fig. 8. The free energy for the formation of the secondary structure and the location of the $3^{\prime}$ end of the short transcripts with respect to the double-stranded region of the RNA are comparable to these features of the predicted secondary structures of minute virus of mice (1), c-myc (10), simian virus 40 early transcription (14), and adenovirus late transcription (25), where transcription pausing or attenuation has been previously described. We are currently inquiring whether the transcript attains the predicted structure in vivo and, if so, the role of the secondary structure in transcription termination.

We have previously shown that transcriptional arrest plays an important role in the tissue-specific expression of the ADA gene (8). The results presented in this communication identify a short sequence important for transcriptional arrest near the $3^{\prime}$ end of the first exon of the murine ADA gene in injected oocytes. Future experiments will explore the contribution of the identified regions of the ADA gene in different contexts of transcriptional controls and tissue- and development-specific expression of the ADA gene.

\section{ACKNOWLEDGMENTS}

We thank Jeffrey Innis, Jeffrey Chinsky, and Randy Mifflin for many fruitful discussions, mouse tissue RNA, and critical comments on the manuscript.

This work was supported by Public Service Health grants GM30204 and AI-25255 from the National Institutes of Health, a grant from Eli Lilly \& Co. to R.E.K., and Public Health Service grant CA-16672 from the National Cancer Institute to D.A.W. V.R. was supported by a postdoctoral fellowship (Q-893) from the Robert A. Welch Foundation.

\section{LITERATURE CITED}

1. Ben-Asher, E., and Y. Aloni. 1984. Transcription of minute virus of mice, an autonomous parvovirus, may be regulated by attenuation. J. Virol. 52:266-276. 
2. Bender, T., C. Thompson, and W. Kuehl. 1987. Differential expression of $c-m y b$ mRNA in murine B lymphomas by a block to transcription elongation. Science 237:1473-1476.

3. Bengal, E., and Y. Aloni. 1989. A block to transcription elongation by RNA polymerase II at synthetic sites in vitro. J. Biol. Chem. 264:9791-9798.

4. Bentley, D. L., and M. Groudine. 1986. A block to elongation is largely responsible for decreased transcription of $c-m y c$ in differentiated HL60 cells. Nature (London) 321:702-706.

5. Bentley, D. L., and M. Groudine. 1988. Sequence requirements for premature termination of transcription in the human $c-m y c$ gene. Cell 53:245-256.

6. Birnstiel, M. L., M. Busslinger, and K. Strub. 1985. Transcription termination and $3^{\prime}$ processing: the end is in site. Cell 41:349-359.

7. Brady, T. G., and C. I. O'Donovan. 1965. A study of the tissue distribution of adenosine deaminase in six mammal species. Comp. Biochem. Physiol. 14:101-102.

8. Chinsky, J. M., M.-C. Maa, V. Ramamurthy, and R. E. Kellems. 1989. Adenosine deaminase gene expression: tissue dependent regulation of transcriptional elongation. J. Biol. Chem. 264: 14561-14565.

9. Chomczynski, P., and N. Sacchi. 1987. Single-step method for RNA isolation by acid guanidinium thiocyanate-phenol-chloroform extraction. Anal. Biochem. 162:156-159.

10. Eick, D., and G. Bornkamm. 1986. Transcriptional arrest within the first exon is a fast control mechanism in $c-m y c$ gene expression. Nucleic Acids Res. 14:8331-8346.

11. Fort, P., A. Vie, M. Piechaczyk, A. Bonnieu, P. Jeanteur, and J. M. Blanchard. 1987. Regulation of $c$-fos gene expression in hamster fibroblasts: initiation and elongation of transcription and mRNA degradation. Nucleic Acids Res. 15:5657-5667.

12. Green, M. R., T. Maniatis, and D. A. Melton. 1983. Human beta-globin pre-mRNA synthesized in vitro is accurately spliced in Xenopus oocyte nuclei. Cell 32:681-694.

13. Gurdon, J. B., and M. P. Wickens. 1983. The use of Xenopus oocytes for the expression of cloned genes. Methods Enzymol. 101:370-386.

14. Hay, N., H. Skolnik-David, and Y. Aloni. 1982. Attenuation in the control of SV40 gene expression. Cell 29:183-193.

15. Ingolia, D. E., M. R. Al-Ubaidi, C.-Y. Yeung, H. A. Bigo, D. A. Wright, and R. E. Kellems. 1986. Molecular cloning of the murine adenosine deaminase gene from a genetically enriched source: identification and characterization of the promoter region. Mol. Cell. Biol. 6:4458-4466.

16. Ingolia, D. E., C.-Y. Yeung, I. F. Orengo, M. L. Harrison, E. G. Frayne, F. B. Rudolph, and R. E. Kellems. 1985. Purification and characterization of adenosine deaminase from a genetically enriched mouse cell line. J. Biol. Chem. 260:13261-13267.

17. Jakobovits, A., D. H. Smith, E. B. Jakobovits, and D. J. Capon. 1988. A discrete element $3^{\prime}$ of human immunodeficiency virus 1 (HIV-1) and HIV-2 mRNA initiation sites mediates transcriptional activation by an HIV trans activator. Mol. Cell. Biol. 8:2555-2561.

18. Kao, S.-Y., A. F. Colman, P. A. Luciw, and B. M. Peterlin. 1987. Antitermination of transcription within the long terminal repeat of HIV-1 by Tat gene product. Nature (London) 330:489-493.

19. Kessler, M., E. Ben-Asher, and Y. Aloni. 1989. Elements mod- ulating the block of transcription elongation at the adenovirus 2 attenuation site. J. Biol. Chem. 264:9785-9790.

20. Kuhn, A., and I. Grummt. 1989. 3'-End formation of mouse pre-rRNA involves both transcription termination and a specific processing reaction. Genes Dev. 3:224-231.

21. Lattier, D. L., J. C. States, J. J. Hutton, and D. A. Wiginton. 1989. Cell type-specific transcriptional regulation of the human deaminase gene. Nucleic Acids Res. 17:1061-1076.

22. Maderious, A., and S. Chen-Kiang. 1984. Pausing and premature termination of human RNA polymerase II during transcription of adenovirus in vivo and in vitro. Proc. Natl. Acad. Sci. USA 81:5931-5935.

23. Manley, J. L. 1988. Polyadenylation of mRNA precursors. Biochim. Biophys. Acta 950:1-12.

24. Melton, D. A., P. A. Krieg, M. R. Rebagliati, T. Maniatis, K. Zinn, and M. R. Green. 1984. Efficient in vitro synthesis of biologically active RNA and RNA hybridization probes from plasmids containing a bacteriophage SP6 promoter. Nucleic Acids Res. 12:7035-7056.

25. Mok, M., A. Maderious, and S. Chen-Kiang. 1984. Premature termination of human RNA polymerase II occurs temporally in adenovirus major late transcriptional unit. Mol. Cell. Biol. 4:2031-2040.

26. Nepveu, A., K. B. Marcu, A. I. Skoultchi, and H. M. Lachman. 1987. Contributions of transcriptional and post-transcriptional mechanisms to the regulation of $c-m y c$ expression in mouse erythroleukemia cells. Genes Dev. 1:938-945.

27. Platt, T. 1986. Transcription termination and the regulation of gene expression. Annu. Rev. Biochem. 55:339-372.

28. Resnekov, O., and Y. Aloni. 1989. RNA polymerase II is capable of pausing and prematurely terminating transcription at a precise location in vivo and in vitro. Proc. Natl. Acad. Sci. USA 86:12-16.

29. Resnekov, O., M. Kessler, and Y. Aloni. 1989. RNA secondary structure is an integral part of the in vitro mechanism of attenuation in simian virus 40. J. Biol. Chem. 264:9953-9959.

30. Selby, M. J., E. S. Bain, P. A. Luciw, and B. M. Peterlin. 1989. Structure, sequence, and position of the stem-loop in tar determine transcriptional elongation by tat through the HIV-1 long terminal repeat. Genes Dev. 3:547-558.

31. Skarnes, W. C., D. C Tessier, and N. H. Acheson. 1988. RNA polymerases stall and/or prematurely terminate nearby both early and late promoters of polyomavirus DNA. J. Mol. Biol. 203:153-171.

32. Watson, R. J. 1988. A transcriptional arrest mechanism involved in controlling constitutive levels of mouse $c-m y b$ mRNA. Oncogene 2:267-272.

33. Wright, S., and J. M. Bishop. 1989. DNA sequences that mediate attenuation of transcription from the mouse protooncogene myc. Proc. Natl. Acad. Sci. USA 86:505-509.

34. Xing, Y. Y., and A. Worcel. 1989. A 3' exonuclease activity degrades the pseudogene 5S RNA transcript and processes the major oocyte 5S RNA transcript in Xenopus oocytes. Genes Dev. 3:1008-1018.

35. Yanofsky, C. 1988. Transcription attenuation. J. Biol. Chem. 263:609-612.

36. Zuker, M., and P. Stiegler. 1981. Optimal computer folding of large RNA sequence using thermodynamics and auxiliary information. Nucleic Acids Res. 9:133-148. 